\title{
PRODUCTIVIDAD DEL HONGO NATIVO Cortinarius austroturmalis EN BOSQUES DE Nothofagus siempreverde y CADUCIFOLIO DE LAS RESERVAS NACIONALES ALTOS DE LIRCAY Y LOS RUILES DE LA VII REGIÓN, CHILE
}

(Productivity of the native mushroom Cortinarius austroturmalis in evergreen and deciduous Nothofagus forests of the National Reserves Altos de Lircay and Los Ruiles of the VII Region, Chile)

\author{
Viviana Salazar V. ${ }^{1^{*}}$, Götz Palfner ${ }^{1}$ \\ 1Laboratorio de Micología y Micorrizas, \\ Facultad de Ciencias Naturales y Oceanográficas, Universidad de Concepción. \\ Víctor Lamas 1290. Concepción. Chile. Teléfono: (56) 41- 2221569 \\ *Autor para correspondencia: vivianasalazar@udec.cl \\ RECIBIDO: 18 de Diciembre de 2015 \\ APROBADO:30 de Diciembre de 2015
}

LOS AUTORES DECLARAN NO TENER CONFLICTO DE INTERESES

Palabras clave: Cortinarius austroturmalis, Chile, Productividad, Reservas, VII Región.

Keywords: Cortinarius austroturmalis, Chile, Productivity, Reserves, VII Region.

\section{RESUMEN}

Los bosques de Nothofagus de la zona mediterránea de Chile, que incluye la VII Región, están constituidos por especies caducifolias y siempreverdes, las cuales se encuentran asociadas a diversos hongos ectomicorrícicos dentro de los que destaca el hongo nativo Cortinarius austroturmalis Moser \& Horak (Agaricales, Basidiomycota). Este hongo constituye un Producto Forestal No Maderero (PFNM) poco conocido, cuya productividad no ha sido determinada, lo que implica que la población rural cercana a las Reservas Nacionales Altos de Lircay y Los Ruiles de la VII Región, no lo identifique como un hongo apto para el consumo humano $\mathrm{y}$, por consiguiente, no lo coseche.

El objetivo de esta investigación fue conocer la productividad de basidiomas del hongo nativo Cortinarius austroturmalis asociado a especies de Nothofagus siempreverdes y caducifolias en las Reservas Nacionales Altos de Lircay y Los Ruiles de la VII Región. Las hipótesis propuestas fueron: 1) La productividad de basidiomas de Cortinarius austroturmalis en la Reserva Nacional Los Ruiles es mayor que en la Reserva Nacional Altos de Lircay, 2) La productividad de basidiomas de Cortinarius austroturmalis asociados a especies de Nothofagus siempreverdes es mayor que la productividad de este hongo asociado a especies de Nothofagus caducifolias en ambas reservas estudiadas.

\footnotetext{
ABSTRACT

Nothofagus forests in the Mediterranean region
} 
of Chile, including VII Region consist of deciduous and evergreen species, which are associated with different ectomycorrhizal fungi within which highlights the native mushroom Cortinarius austroturmalis Moser \& Horak (Agaricales, Basidiomycota). This fungus is a Non-Wood Forest Product (NWFP) obscure, whose productivity has not been determined, implying that close to the National Reserves Altos de Lircay and Los Ruiles, VII Region, rural population does not identify it a fungus suitable for human consumption and therefore not harvested.

The objective of this research was to determine the productivity of fruit body of the native mushroom Cortinarius austroturmalis associated with Nothofagus species of evergreen and deciduous in the National Reserves Altos de Lircay and Los Ruiles, VII Region. The proposed hypotheses were: 1) Productivity of Cortinarius austroturmalis in the National Reserve Los Ruiles is higher than in the National Reserve Altos de Lircay, 2) Productivity of Cortinarius austroturmalis associated with Nothofagus species evergreen is greater than the productivity of this fungus associated with deciduous Nothofagus species in both reserves studied.

\section{INTRODUCCIÓN}

Desde los comienzos de nuestra historia, la recolección de productos forestales no madereros (PFNM) asociados a los bosques se ha ido desarrollando, tanto por los pueblos originarios como por las comunidades rurales, pero su estudio e investigación son muy recientes. Los PFNM son aquellos bienes de origen biológico distinto de la madera, procedentes de los bosques, de otros terrenos arbolados y de árboles situados fuera de los bosques, considerando bienes de origen animal y vegetal, independiente de la naturaleza artificial o natural del bosque (INFOR, 2009). Los hongos comestibles conocidos en nuestro país son pocos, pero muchas especies nativas tienen potencial de PFNM y los criterios que comparten son: distribución am- plia, abundancia, características organolépticas atractivas, fácilmente reconocibles y riesgo bajo de confusión. Dentro de los hongos considerados PFNM relevantes en Chile, están aquellos distribuidos en los bosques de Nothofagus de la VII Región de nuestro país, donde junto a hongos parásitos biotróficos como el llao llao (Cyttaria hariotii E. Fisch.), se describe la presencia de especies micorrícicas como el hongo nativo Cortinarius austroturmalis que produce setas comestibles poco conocidas por las comunidades aledañas (Garrido, 1988; Lonsdale et al., 2008; Brundrett, 1991).

En nuestro país se han registrado más de 3.000 especies de hongos (Mujica \& Vergara, 1980; Moser \& Horak, 1975; Garrido, 1986; 1988), de las cuales se han descrito como comestibles al menos 15 especies nativas recolectadas por el pueblo mapuche, principalmente, en otoño e invierno (López \& Fuenzalida, 1998). Los hongos silvestres comestibles son importantes, porque proporcionan beneficios fundamentales para la población: son fuentes de alimento, de beneficios económicos y algunas especies comestibles tienen propiedades medicinales (Reshetnikov et al.,2001). Algunas de las especies de hongos silvestres comestibles que habitan en Chile son: Morchella conica Pers., Ramaria flava (Schaeff.) Quél., Ramaria botrytis (Pers.) Ricken, Ramaria subaurantiaca Corner, Boletus loyo Phillippi, Boletus loyita E. Horak, Cortinarius lebre Garrido y Cyttaria espinosae Lloyd, entre otros (FIA, 1996).

En la mayoría de los casos, los hongos chilenos se encuentran estrechamente asociados a especies vegetales nativas como árboles del género Nothofagus mediante una asociación obligatoria denominada ectomicorriza, donde el micelio invade la raíz sin entrar en el interior de las células (Garrido, 1988; Palfner, 2001). Para Chile y Argentina, Moser \& Horak (1975) fueron quienes advirtieron la asociación obligatoria de las especies del género Cortinarius a árboles de Nothofagus y en las reservas estudiadas en la VII Región, el hongo nativo Cortinarius austroturmalis se asocia a 
árboles como: Nothofagus obliqua (Mirb.) Oerst. (Hualle), Nothofagus dombeyi (Mirb.) Oerst. (Coigüe), Nothofagus alessandrii Espinosa (Ruíl) y Nothofagus glauca (Phil.) Krasser (Hualo) (Rodríguez et al., 2005). Según un estudio realizado en la Cordillera Pelada de la X Región de Chile por Valenzuela et al. (1998), el género Cortinarius presenta una mayor cantidad de especies asociadas a Nothofagus dombeyi y Nothofagus obliqua, lo que coincide con Singer \& Moser (1965) y otras investigaciones hechas previamente en nuestro país. De lo anterior, se deduce que los bosques de Nothofagus, suelen ser los hábitats preferidos por la mayoría de las especies del género Cortinarius que habitan en Chile, incluyendo la especie estudiada, Cortinarius austroturmalis.

A pesar que C. austroturmalis ha sido registrado en nuestro país entre la zona Centro sur y Magallanes, incluyendo áreas colindantes de Argentina y su comestibilidad ha sido comprobada (Moser \& Horak, 1975), no hay información sobre su productividad o calidad como alimento.

Ecológicamente, la productividad es un parámetro necesario de medir, pues constituye una característica importante de las poblaciones que sirve como índice para definir el funcionamiento de cualquier ecosistema y su estudio puede hacerse a nivel de especies, cuando interesa su aprovechamiento económico (Lugo \& Morris, 1982). Pilz \& Molina (2002), definen la productividad biológica de los hongos como el número total o el peso de setas por unidad de área en el transcurso de una temporada de fructificación (formación de carpóforos). El objetivo de esta investigación fue conocer la productividad de basidiomas del hongo nativo Cortinarius austroturmalis asociado a especies de Nothofagus siempreverdes y caducifolias en las Reservas Nacionales Altos de Lircay y Los Ruiles de la VII Región.

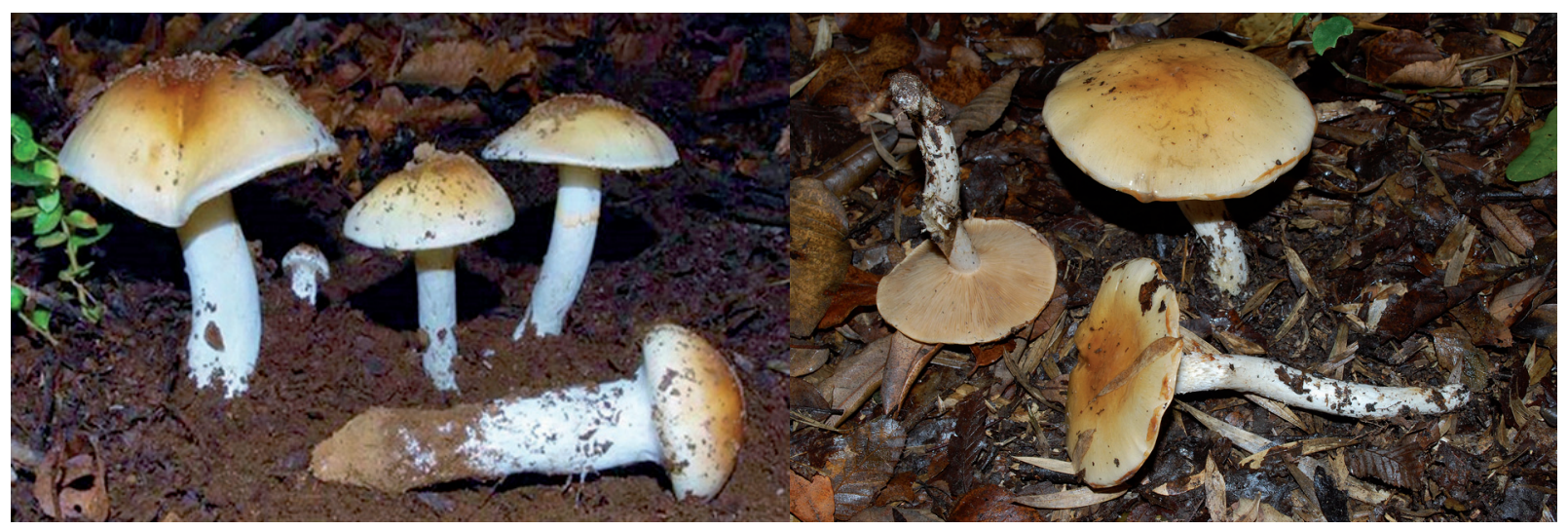

Figura 1. Cortinarius austroturmalis.

\section{MATERIALES Y MÉTODOS}

\section{Áreas de estudio:}

\section{Reserva Nacional Altos de Lircay}

\section{Ubicación}

El trabajo en terreno se realizó durante la temporada otoño-invierno en la Reserva Altos de Lircay (Fig. 2), situada en la Precordillera de los Andes, comuna de San Clemente, VII Región, entre $\operatorname{los} 35^{\circ} 32^{\prime}$ a $35^{\circ} 40^{\prime}$ de latitud Sur y los meridianos $70^{\circ} 50^{\prime}$ a $71^{\circ} 03^{\prime}$ de longitud Oeste.

\section{Condiciones climáticas}

El clima de esta reserva es de tipo templado cálido con una estación seca prolongada. En algunas áreas ubicadas hacia el interior se dan algunas características de clima de montaña debido a la mayor altitud, con presencia de nevazones frecuentes que hacen que la nieve permanezca en el sector oriente durante el invierno y parte de la prima- 
vera, especialmente en las laderas montañosas de exposición Sur y Este. La media anual de temperatura es de $14,7^{\circ} \mathrm{C}$ y el promedio anual de precipitaciones alcanzan a $980 \mathrm{~mm}$ (CONAF 2014).

\section{Reserva Nacional Los Ruiles}

\section{Ubicación}

También, se realizó un trabajo en terreno en la Reserva Los Ruiles (Fig. 2), ubicada en el término municipal de Pelluhue, en la Provincia de Cauquenes, VII Región, entre los $35^{\circ} 36^{\prime}$ de latitud Sur y los meridianos $72^{\circ} 21^{\prime}$ de longitud Oeste, durante el mismo tiempo.

\section{Condiciones climáticas}

La reserva posee un clima del tipo templadocálido con periodos estivales secos e invernales lluviosos, con sequía estival de 4 meses como mínimo. La temperatura media anual es de $13,8^{\circ} \mathrm{C}$ y las precipitaciones de 774,45 mm (CONAF 2014).

\section{Establecimiento de parcelas en las reservas en estudio}

Dentro de la Reserva Altos de Lircay se establecieron 24 parcelas de $20 \times 20 \mathrm{~m}$ en el verano del año 2013, 12 dominadas por Nothofagus obliqua y 12 dominadas por Nothofagus dombeyi, situadas aproximadamente a $1500 \mathrm{msnm}$, todas con exposición noroeste. Estas parcelas se recorrieron en busca del hongo comestible Cortinarius austroturmalis durante la temporada otoño- invierno.

En la Reserva los Ruiles se establecieron nueve parcelas de 20 × 20 m, tres dominadas por Nothofagus alessandrii, tres dominadas por Nothofagus glauca y tres dominadas por $N$. dombeyi en el verano del año 2014, situadas a una altura de 200-400 msnm aproximadamente, todas con exposición sur. Estas parcelas también se recorrieron durante la temporada otoñoinvierno cosechando el hongo C. austroturmalis.

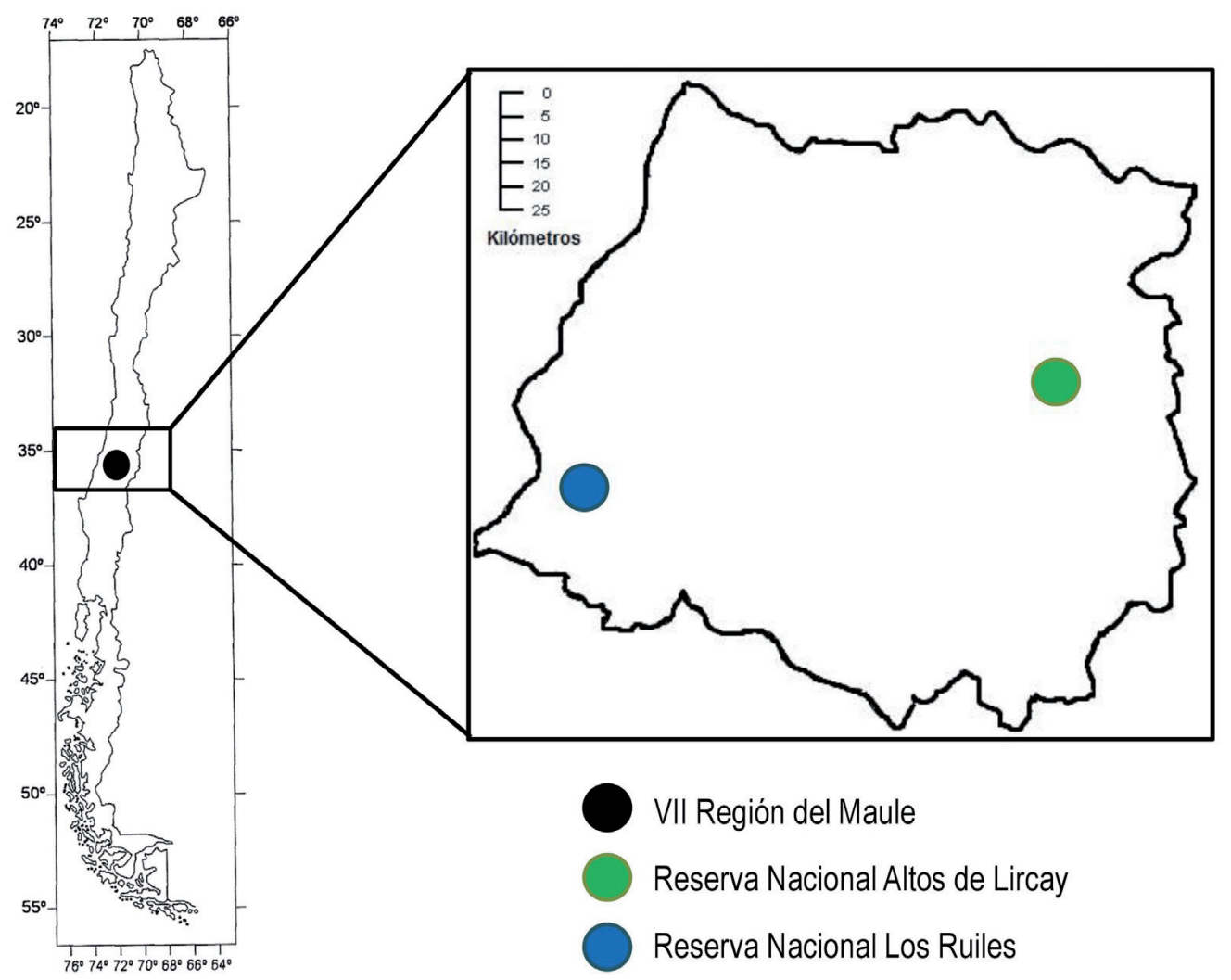

Figura 2. Mapa de la ubicación de las Reservas Nacionales Altos de Lircay y Los Ruiles. 


\section{Recolección, documentación e identificación}

Se realizaron cuatro muestreos en distintas fechas y en distintos años para cada parcela demarcada en cada una de las reservas durante la temporada otoño-invierno, con enfoque en la especie comestible $C$. austroturmalis. Los cuerpos fructíferos recolectados fueron documentados en terreno mediante fotografías digitales y la toma de datos referenciales usando un GPS antes de ser llevados en contenedores plásticos al laboratorio.

Los ejemplares de $C$. austroturmalis recolectados fueron identificados en terreno mediante sus características macromorfológicas $\mathrm{y}$, posteriormente, en el laboratorio se confirmó la identidad de la especie mediante la observación de sus esporas a través de microscopía óptica y se midió la biomasa fresca de cada colección en kilogramos en una balanza portátil. Después se deshidrataron las setas en una estufa de secado ventilada a $50^{\circ} \mathrm{C}$ durante 48 h. Finalmente, se seleccionaron muestras de referencia que fueron depositadas en la colección de hongos del Herbario de la Universidad de Concepción (Fungario CONC-F).

\section{Comparación de la productividad de basidio-} mas de $C$. austroturmalis recolectados en dos Reservas de la VII Región

La productividad del hongo en estudio, se determinó midiendo las variaciones de producción por unidad de tiempo y espacio según el muestreo descrito en los puntos anteriores para ambas reservas incluidas en esta investigación, lo que se hizo considerando la biomasa seca de los basidiomas recolectados de C. austroturmalis, para luego mediante un factor de conversión (biomasa seca-fresca) obtener el peso fresco del hongo (El factor de conversión calculado para C. austroturmalis es 11,85$)$.

Se determinó la productividad en $\mathrm{g} / \mathrm{ha}$ de $C$. austroturmalis en la Reserva Nacional Altos de Lircay en el año 2013 para, posteriormente, estimar la productividad del mismo hongo en la Reserva Nacional Los Ruiles durante la temporada otoñoinvierno del 2014. Una vez determinada la produc- tividad en cada una de las reservas consideradas en esta investigación, se procedió a comparar la productividad del hongo comestible $C$. austroturmalis entre ellas y, a continuación, entre las especies de Nothofagus siempreverdes y caducifolias presentes en ambas reservas, con el fin de determinar cuál de las dos poseía una mayor cantidad de este recurso.

\section{RESULTADOS}

Los datos sobre productividad presentados a continuación, han sido recogidos durante la temporada otoño-invierno de los años 2013 y 2014. El hongo en estudio $C$. austroturmalis, fue encontrado en la mayoría de las parcelas establecidas en ambas reservas incluidas en esta investigación.

Productividad en biomasa seca y fresca de $C$. austroturmalis en las Reservas Nacionales Altos de Lircay y Los Ruiles

La productividad estimada en biomasa seca del hongo $C$. austroturmalis fue menor para la Reserva Nacional Altos de Lircay (412 g/ha) en comparación con la obtenida para la Reserva Nacional Los Ruiles (588 g/ha). La productividad estimada en biomasa fresca del hongo $C$. austroturmalis fue menor para la Reserva Nacional Altos de Lircay (4.882 g/ha) en comparación con la obtenida para la Reserva Nacional Los Ruiles (6.968 g/ha) (Fig. 3).

Productividad de $C$. austroturmalis en biomasa seca $y$ fresca asociada a las distintas especies de Nothofagus de la Reserva Altos de Lircay

La productividad de $C$. austroturmalis en la Reserva Altos de Lircay fue más alta en parcelas dominadas por la especie siempreverde Nothofagus dombeyi, donde este hongo fue encontrado con un promedio de biomasa seca de $800 \mathrm{~g} / \mathrm{ha}(97 \%$ del total de la reserva), contrastado con sólo $24 \mathrm{~g} /$ ha $(3 \%$ del total de la reserva) como promedio de biomasa seca en parcelas dominadas por la especie caducifolia Nothofagus obliqua. La productividad de C. austroturmalis en la Reserva Altos de Lircay 


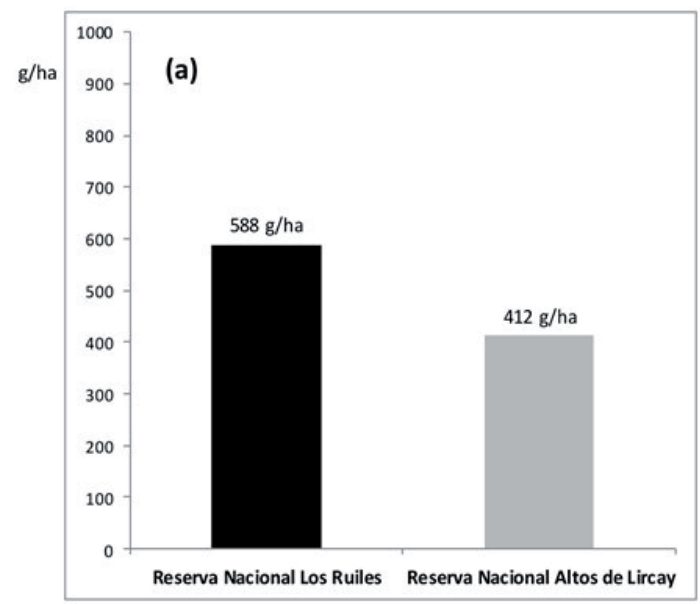

Figura 3. Diferencia en $\mathrm{g} / \mathrm{ha}$ de la productividad en Cortinarius austroturmalis entre las reservas en estudio.

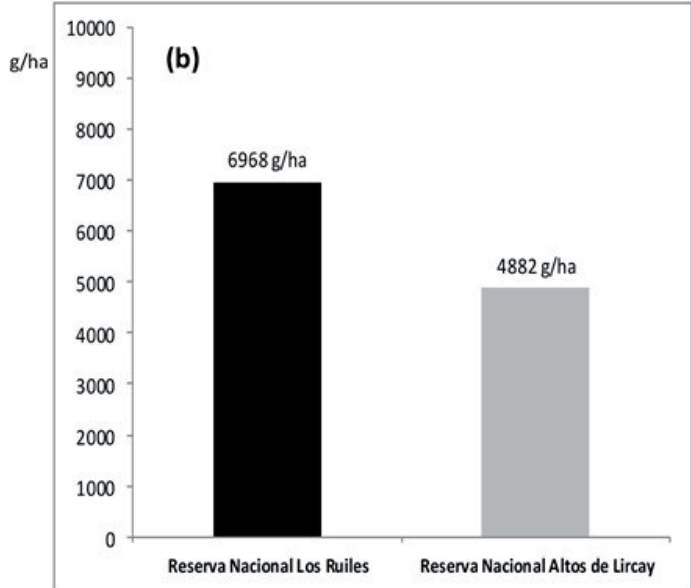

(a) biomasa seca y (b) biomasa fresca del hongo fue más alta en parcelas dominadas por Nothofagus dombeyi, donde este hongo fue encontrado con un promedio de biomasa fresca de $9.480 \mathrm{~g} / \mathrm{ha}(97 \% \mathrm{del}$ total de la reserva), contrastado con $284 \mathrm{~g} / \mathrm{ha}$ ( $3 \%$ del total de la reserva) como promedio de biomasa fresca en parcelas dominadas por la especie caducifolia Nothofagus obliqua (Fig. 4).

Productividad de $C$. austroturmalis en biomasa seca $y$ fresca asociada a las distintas especies de Nothofagus de la Reserva Los Ruiles

El promedios de biomasa seca de C. austrotur- malis asociados a las tres especies de Nothofagus predominantes en las parcelas establecidas, fue más alta en parcelas dominadas por la especie caducifolia Nothofagus alessandrii, con 966 g/ha (55\% del total de la reserva), seguida por la productividad del hongo asociado a la especie siempreverde $\mathrm{No}$ thofagus dombeyi con $484 \mathrm{~g} / \mathrm{ha}$ ( $27 \%$ del total de la reserva) y finalmente, la menor productividad de C. austroturmalis se observó asociada a la especie caducifolia Nothofagus glauca con 315 g/ha (18\% del total de la reserva). Respecto a la biomasa fresca la productividad de $C$. austroturmalis en la Reserva
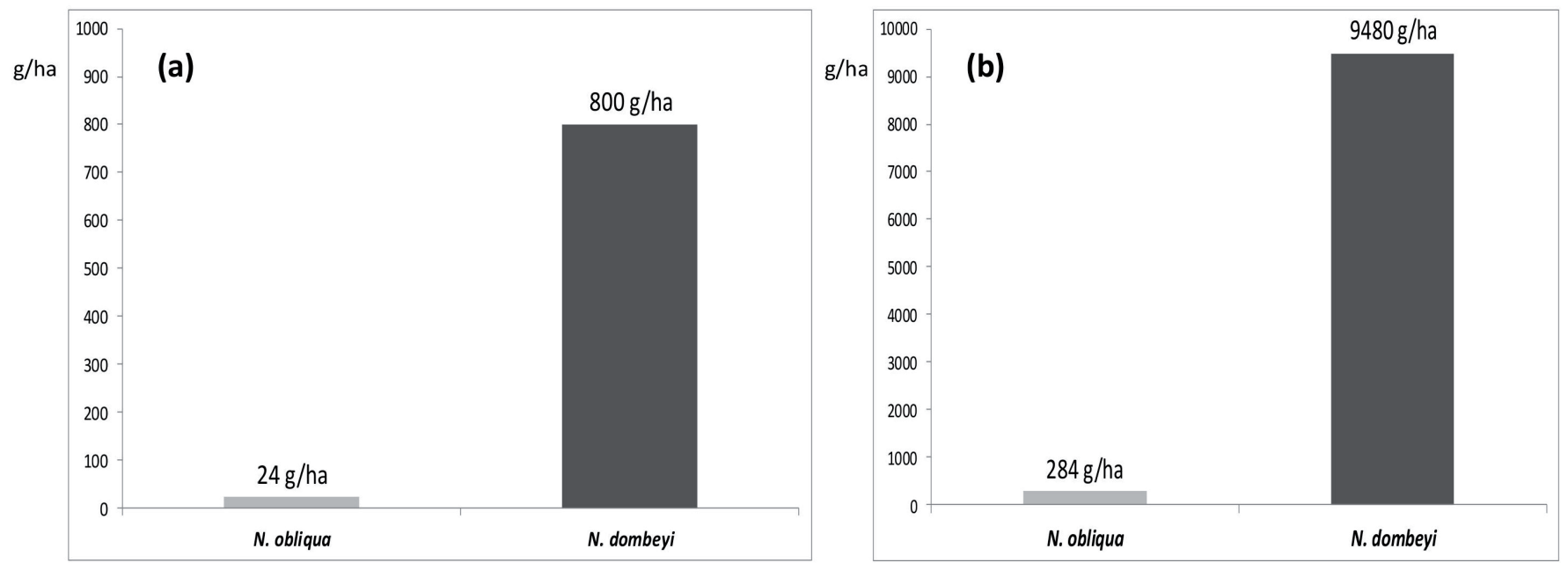

Figura 4. Productividad en (a) biomasa seca y (b) biomasa fresca de Cortinarius austroturmalis en g/ ha asociada a especies de Nothofagus caducifolias y siempreverdes en Altos de Lircay. 
Los Ruiles fue más alta en parcelas dominadas por Nothofagus alessandrii, con un promedio de biomasa fresca del hongo estudiado de $11.447 \mathrm{~g} / \mathrm{ha}(97 \%$ del total de la reserva), contrastado con $5.735 \mathrm{~g} / \mathrm{ha}$

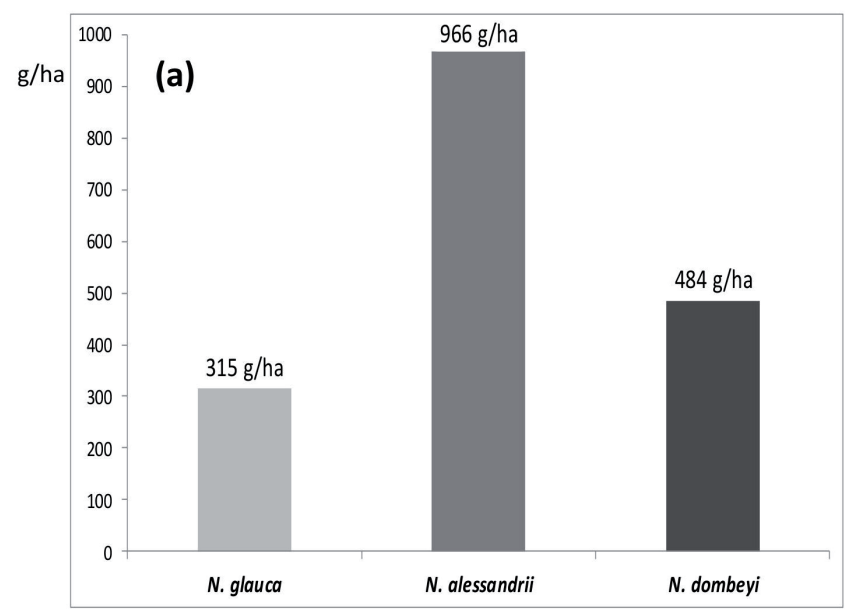

( $3 \%$ del total de la reserva) como promedio de biomasa fresca en parcelas dominadas por la especie siempreverde Nothofagus dombeyi (Fig. 5).

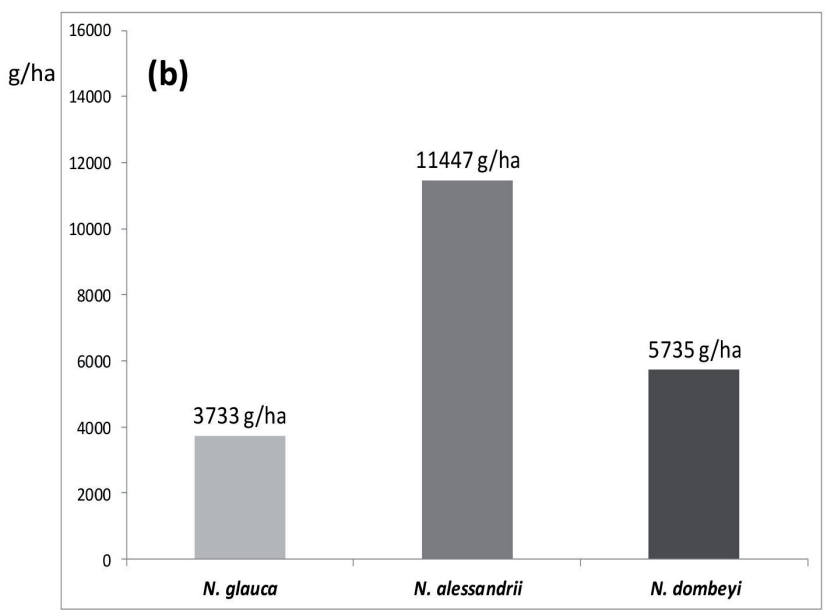

Figura 5. Productividad en (a) biomasa seca y (b) biomasa fresca de Cortinarius austroturmalis en g/ ha asociada a especies de Nothofagus caducifolias y siempreverdes en Los Ruiles.

\section{DISCUSIÓN}

En Chile se ha masificado cada vez más el estudio de los hongos comestibles cultivables como una buena fuente de ingresos económicos, sin embargo, no se han hecho estudios sobre la productividad de hongos silvestres comestibles en Reservas Nacionales, por lo que en este trabajo se decidió estudiar la productividad del hongo Cortinarius austroturmalis, por ser éste una especie comestible (Moser \& Horak, 1975) y además, el hongo más frecuente y abundante en las reservas estudiadas de la VII Región. En este estudio se obtuvo que la productividad (en biomasa seca) de C. austroturmalis en la Reserva Nacional Altos de Lircay (412 g/ha) fue menor en un 18\% que la obtenida en la Reserva Nacional Los Ruiles (588 g/ ha), por lo que se acepta la Hipótesis 1 que afirma que la productividad de basidiomas de C. austroturmalis en la Reserva Nacional Los Ruiles es mayor que en la Reserva Nacional Altos de Lircay. Lo anterior puede deberse a varios factores, como por ejemplo, el número de parcelas consideradas para cada reserva, ya que en la Reserva Altos de Lircay se consideraron 24 parcelas en total (12 dominadas por $N$. obliqua y 12 dominadas por $N$. dombeyi), mientras que en la Reserva Los Ruiles sólo nueve parcelas. Además, las condiciones climáticas predominantes en cada una de las reservas consideradas también pudieron influir en los resultados de productividad obtenidos, ya que si bien los promedios anuales de temperatura y precipitación son similares para ambas reservas, en la Reserva Altos de Lircay la mayor altitud topográfica (1500 $\mathrm{msnm}$ ) provoca más días con heladas que en la Reserva Los Ruiles (200-400 msnm) y una fluctuación más amplia entre temperaturas máximas y mínimas con diferencias hasta $20^{\circ} \mathrm{C}$ entre noche y día (el promedio de la temperatura mínima en julio, mes que marca la mitad de la temporada de fructificación, es $<0^{\circ} \mathrm{C}$ en Altos de Lircay y entre 5 y $9^{\circ} \mathrm{C}$ en Los Ruiles), debido a que la última zona se encuentra bajo influencia marítima y a una altitud no mayor que $400 \mathrm{msnm}$. Sin embargo, se sabe que los buenos o malos años de producción de setas no se explican sólo por los factores climáti- 
cos predominantes, sino también por otros factores como la propia biología del hongo (Straatsmaa et al., 2001).

En esta investigación sólo se consideró una temporada de recolección de cuerpos fructíferos para cada reserva, por motivos de tiempo, por lo que se deberían hacer inventarios repetidos (vigilancia) durante varias temporadas de fructificación para estimar adecuadamente la productividad del área, ya que existe una variación anual considerable en la productividad entre las temporadas de fructificación que suele relacionarse con el clima (Vogt et al.,1992; Liegel, 1998; Pilz et al., 1999).

En la Reserva Altos de Lircay se observó una mayor productividad del hongo en estudio (en biomasa seca) asociada a la especie siempreverde Nothofagus dombeyi (800 g/ha) en contraposición con la especie caducifolia $N$. obliqua $(24 \mathrm{~g} /$ ha), mientras que para la Reserva Los Ruiles se observó que la mayor productividad del hongo en estudio se dio asociada a la especie caducifolia $N$. alessandrii (966 g/ha), seguida por $N$. dombeyi (484 g/ha) y N. glauca (315 g/ha). Debido a lo expuesto anteriormente, se acepta parcialmente la Hipótesis 2 que dice que la productividad de basidiomas de $C$. austroturmalis asociados a especies de Nothofagus siempreverdes es mayor que la productividad de este hongo asociado a especies de Nothofagus caducifolias en ambas reservas estudiadas. La mayoría de las setas comestibles de los bosques se asocian a los árboles mediante micorrizas y dependen de los carbohidratos producidos éstos (Egli et al., 2010), por lo que se entiende que en la Reserva Altos de Lircay el hongo C. austroturmalis eligió asociarse mayormente a la especie siempreverde $N$. dombeyi, porque ésta en días de sol le proveía sombra con sus hojas, mantenía la humedad del suelo, protegiéndolo contra la evapotranspiración y le aportaba los nutrientes necesarios para el crecimiento de sus cuerpos fructíferos, condiciones que la especie caducifolia $N$. obliqua no podía aportarle durante la temporada otoño-invierno, donde se encontraba sin hojas, pues se ha demostrado que los hongos formadores de micorrizas dependen de los fotosintatos para las actividades de mantenimiento y de producción de setas (Hacskaylo,1965; Högberg et al., 2001). Una reducción o una interrupción del flujo de hidratos de carbono desde las hojas del árbol hacia las raíces afecta la micorrización y la producción de cuerpos fructíferos, por lo tanto, la eliminación de la parte verde activa del árbol conduce a una interrupción inmediata de la fotosíntesis y formación de setas ectomicorrícicas (Kropp \& Albee, 1996; Ohenoja, 1988).

En el caso de la Reserva Los Ruiles, el crecimiento de $C$. austroturmalis se vio favorecido asociado a la especie caducifolia $N$. alessandrii por estar ubicada en lugares más húmedos que $N$. dombeyi y $N$. glauca, con bastante sombra para mantener el suelo húmedo en comparación con el suelo seco predominante para el resto de la reserva. En definitiva, la productividad de C. austroturmalis en ambas reservas, se vio beneficiada principalmente por las condiciones de humedad presente en el suelo.

Para saber si la productividad promedio de $C$. austroturmalis obtenida en peso fresco para las reservas estudiadas en la VII Región es significativa con respecto a otros hongos chilenos recolectados, se destaca lo indicado por Garfias et al. (1995) que señala que en Chile los principales hongos comestibles producidos corresponden a las especies Suillus luteus y Lactarius quieticolor, constituyendo más del $90 \%$ de las exportaciones de hongos. Estos hongos se desarrollan en las plantaciones de Pinus radiata de 6 a 20 años, con rendimientos de $300 \mathrm{~kg} / \mathrm{ha} /$ año en biomasa fresca. Al comparar este valor con los resultados obtenidos en promedio de biomasa fresca para C. austroturmalis en ambas reservas estudiadas $(9 \mathrm{~kg} / \mathrm{ha} /$ año), podemos observar que existe una significativa diferencia en cuanto a la cantidad producida en $\mathrm{kg} / \mathrm{ha} / \mathrm{año}$, la que se debe a que en las plantaciones de pino hay poca diversidad $y$, por lo tanto, poca competencia para el desarrollo de basidiomas de 
las especies comestibles mencionadas, lo que permite que la productividad de estas setas sea muy elevada. Además, la recolección de setas se realiza durante toda la temporada de fructificación, no sólo efectuando muestreos aislados cada cierto intervalo de tiempo como es el caso de este estudio.

Existen pocos valores publicados sobre productividad de hongos en países distintos a Chile. Slee (1991), sobre la base de observaciones personales, estimó que en algunos bosques escoceses la productividad de chantarelas (Cantharellus cibarius Fr.), reconocidas a nivel mundial como una de las mejores setas comestibles de los bosques, alcanza $50 \mathrm{~kg} / \mathrm{ha} / \mathrm{año}$. En el proyecto MAB de chantarelas, realizado en el noroeste del Pacífico, EE.UU., se observó una productividad con valores entre 0,076 a $21,947 \mathrm{~kg} / \mathrm{ha} / \mathrm{año}$, con un promedio de $2,520 \mathrm{~kg} /$ ha/año, reportado como peso fresco (Pilz et al., 2003). Como se indicó anteriormente, la productividad promedio en peso fresco de C. austroturmalis para las reservas estudiadas de la VII Región alcanzó un valor de $9 \mathrm{~kg} / \mathrm{ha} / \mathrm{año}$, que comparado con el estudio hecho por Pilz et al. (2003), se convierte en una cifra que nos permite considerar a este hongo nativo chileno, como un PFNM apropiado para continuar siendo estudiado en busca de mejores alternativas de recolección, manejo y explotación.

Los hongos del bosque, incluyendo hongos comestibles, contribuyen a la salud de éstos y de las redes alimentarias de varias formas (Carroll \& Wicklow, 1992; Molina et al., 1999; Read et al., 1992), y los administradores deben considerar estos roles y funciones cuando desarrollan instrucciones de recolección o planes de gestión. Dadas las numerosas especies de hongos cosechados, la diversidad de tipos de bosques que habitan, y la amplia gama de sistemas silvícolas forestales que utilizan, existen muchas oportunidades para mejorar la gestión de las setas a través de una mejor comprensión de su biología, ecología, ecosiste- mas y funciones (Pilz \& Molina, 2002). En otros países se han hecho estudios similares al de esta investigación, pero con la finalidad de mejorar el manejo y aprovechamiento de los hongos en los bosques, por ejemplo, Pilz et al. (2004) hicieron un estudio sobre la productividad de las morchelas en lo que se refiere a las actividades de gestión forestal y eventos de perturbación, debido a los grandes cultivos comerciales de morchelas que se cosechan anualmente en los bosques de coníferas de la montaña del noroeste de los Estados Unidos.

\section{CONCLUSIÓN}

En esta investigación, se logró establecer diferencias en la productividad del hongo nativo C. austroturmalis asociado a las Reservas Nacionales Altos de Lircay y Los Ruiles de la VII Región, siendo mayor la productividad de este hongo (en biomasa seca) en la Reserva Los Ruiles con $588 \mathrm{~g} /$ ha en comparación con el resultado obtenido para la Reserva Altos de Lircay de 412 g/a. Destacar que este es el primer estudio hecho en nuestro país sobre productividad de un hongo nativo en Reservas Nacionales, pero queda mucho más por hacer en el futuro, ya sea en otras reservas, parques y bosques de Chile o en las mismas reservas incluidas en esta investigación, pero a largo plazo, ya que al realizar monitoreos sobre la productividad de una o varias especies de hongos en estos lugares, se pueden ir creando nuevas estrategias de manejo de los hongos en el bosque nativo, asegurando el uso sostenible de este recurso no maderero, de modo de poder aprovecharlo al máximo sin dañar el ecosistema. Asimismo, aún quedan tareas por hacer, tales como, análisis bromatológicos que permitan conocer con mayor detalle la composición de C. austroturmalis en cuanto a proteínas, aminoácidos, grasas y otros componentes de importancia desde el punto de vista nutritivo. 


\section{AGRADECIMIENTOS}

Agradecemos al Dr. Patricio López, al Prof. Roberto Rodríguez y a la Dra. Claudia Pérez de la Universidad de Concepción, por la orientación para rea- lizar este trabajo. Esta investigación fue financiada por el proyecto CONAF $\mathrm{N}^{\circ} 065 / 2012$ "Bases para definir estrategias y prácticas de manejo sustentable del bosque nativo, usando el estado de conservación de líquenes, algas y hongos superiores".

\section{REFERENCIAS}

Brundrett, M. (1991). Mycorrhizas in natural ecosystems. In Begon M., Fitter A. H. \& A. Macfadyen (eds.): Advances in Ecological Research, 21:171-313.

Carroll, G. C. \& Wicklow, D. T. (Eds.). (1992). The Fungal Community: Its Organization and Role in the Ecosystem, 2nd Edition. Marcel Dekker, New York, 976 pp.

CONAF. (2014). Plande manejo de la Reserva Nacional Altos de Lircay. URL: http://www.conaf. cl/parques/reserva-nacional-altos-de-lircay/. Revisado: 7 de julio, 2014.

CONAF. (2014). Plande manejo de la Reserva Nacional Los Ruiles. URL: http://www.conaf.cl/ parques/reserva-nacional-los-ruiles/. Revisado: 7 de julio, 2014.

Egli, S.; Ayer, F.; Peter, M.; Eilmann, B.; \& Rigling, A. (2010). Is forest mushroom productivity driven by tree growth? Results from a thinning experiment. Ann For Sci 67 (509): 9.FIA. (1996).

Introducción de nuevas especies de hongos comestibles. Estudio de mercado. Ministerio de la Agricultura. Santiago. Chile. 201 pp.

Garfias, R.; Carmona, R.; Barros, D.; Cabellos, J.A.; \& Baldini, A. (1995). Informe de Países. Chile. Consulta de Expertos sobre Productos Forestales No Madereros para América Latina y el Caribe. Memoria - congreso. FAO/RLC. Santiago. pp: 200 - 208.
Garrido, N. (1986). Survey of ectomycorrhizal fungi associated with exotic forest trees in Chile. Nova Hedwigia 43: 423-442.

Garrido, N. (1988). Agaricales und ihre mykorrhizen in den Nothofagus-Wáldem mittelchiles. Bibliotheca Mycologica tomo 120, J. Cramer, Berlín, Stuttgart, Alemania, 528 pp.

Hacskaylo, E. (1965). Telephora terrestris and mycorrhizae of Virginia pine. For Sci 11:401-404. Högberg, P.; Nordgren, A.; Buchmann, N.; Taylor, A.; Ekblad, A.; Högberg, M.; et al. (2001). Largescale forest girdling shows that current photosynthesis drives soil respiration. Nature 411:789-792

INFOR. (2009). Exportaciones forestales de productos no madereros. Boletín $\mathrm{N}^{\circ} 4$, Septiembre 2009. 18 pp.

Kropp, B. R.; \& Albee, S. (1996). The effects of silvicultural treatments on occurrence of mycorrhizal sporocarps in a Pinus contorta forest: a preliminary study. Biol Conserv 78:313-318

Liegel, L. (Ed.). (1998). The biological, socioeconomic, and managerial aspects of chanterelle mushroom harvesting: the Olympic Peninsula, Washington State, USA AMBIO Special Report No. 9,35 pp.

Lonsdale, D.; Pautasso, M.; \& Holdenrieder, O. (2008). Wood decaying fungi in the forest: conservation needs and management options. European Journal of Forest Research 127: 1-22. 
López, L. \& Fuenzalida, F. (1998). Algunos Problemas Identificados en la Comercialización de Productos Provenientes del Bosque Nativo. Proyecto Manejo Sustentable del Bosque Nativo. CONAF - GTZ.

Lugo, A. \& Morris, G. (1982). Los sistemas ecológicos y la humanidad. Washington D. C., OEA. Secretaria General. 82 pp.

Molina, R.; O'Dell, T.; Dunahm, S.; \& Pilz, D. (1999). Biological diversity and ecosystem functions of forest soil fungi: management implications. In: Meurisse, R.T., Ypsilantis, W.G., Seybold C. (Eds.), Proceedings of the Pacific Northwest Forest and Rangeland Soil Organism Symposium. USDA For. Serv. Gen. Tech. Rep. PNW-461: 45-58.

Moser M. \& Horak E. (1975). Cortinarius Fr. und nahe verwandte Gattungen in Südamerika. Beih. Nova Hedwigia 52: 1-628.

Mujica, F. \& Vergara, C. (1980). Flora fungosa chilena. Segunda Edición. Ciencias Agrícolas, Facultad de Agronomía, Universidad de Chile, Chile 308 pp.

Ohenoja, E. (1988). Behaviour of mycorrhizal fungi in fertilized forest. Karstenia 28: 27-30.

Palfner, G. (2001). Taxonomische studien an ektomykorrhizen aus den Nothofagus-Wãldern Mittelsüdchiles. Bibliotheca Mycologica 190, J. Cramer, Berlin, Stuttgart, Alemania. 243 pp.

Pilz, D.; Smith, J.; Amaranthus, M.; Alexander, S.; Molina, R.; \& Luoma, D. (1999). Managing the commercial harvest of the American matsutake and timber in the southern Oregon Cascade Range. J. For. 97 (2), 8-15.

Pilz, D. \& Molina, R. (2002). Commercial har- vests of edible mushrooms from the forests of the Pacific Northwest United States: issues, management, and monitoring for sustainability. Forest Ecology and Management 155: 3-16.

Pilz, D.; Norvell, L.; Danell, E.; \& Molina, R. (2003). Ecology and mnapmt of cornmercially harvested chanterelle mushrooms. Gen. Tech. Rep. PNW-GTR-576. USDA For. Serv., Pacific Northwest Res. Sta., Portland, OR 83 pp.

Pilz, D.; Weber, N.; Carter, M.; Parks, C.; \& Molina, R. (2004). Productivity and diversity of morel mushrooms in healthy, burned and insectdamaged forests of northeastern Oregon. Forest Ecology and Management 198:367-386.

Read, D.J.; Lewis, D.H.; Fitter, A.H.; \& Alexander, I.J. (1992). Mycorrhizas in Ecosystems. CAB International, Wallingford, UK, 419 pp.

Reshetnikov, S.; Wasser, S.; \& Tan, K. (2001). Higher basidiomycota as a source of Antitumour and immunostimulating polysaccharides. A review. International Journal of Medicinal Mushrooms 3: 361-394.

Rodríguez, R.; Ruíz, E.; \& Elissetche, J. (2005). Árboles en Chile. Editorial Universidad de Concepción. Concepción, Chile. 183 pp.

Singer, R. \& Moser, M. (1965). Forest mycology and forest communities in South America. Mycopathology et Mycology Applied 26: 9-191.

Slee, R. W. (1991). The potential of small woodlands in Britain for edible mushroom production. Scottish Forestry 45: 3-12.

Straatsma, G.; Ayer, F.; \& Egli, S. (2001). Species richness, abundance, and phenology of fungal fruit bodies over 21 years in a Swiss forest plot. Mycol Res 105:515-523. 
Valenzuela, E.; Moreno, G.; Garnica, S.; \& Ra- Vogt, K. A.; Bloomfield, J.; Ammirati, J. F.; \& mírez, C. (1998). Micosociología en bosques nati- Ammirati, S. R. (1992). Sporocarp production by vos de Nothofagus y plantaciones de Pinus radiata en la X Región de Chile: diversidad y rol ecológico. Revista Chilena de Historia Natural 71: 133146. Basidiomycetes, with emphasis on forest ecosystems. In: Carroll, G.C. Wicklow, D.T. (Eds.), The Fungal Community: Its Organization and Role in the Ecosystem, 2nd Edition. Marcel Dekker, New York, NY, pp. 563-581. 